Voix et Images

\title{
Un homme et son péché : l'innocente avarice ou le masque idéologique
}

\section{Alain Piette}

Volume 4, numéro 1, septembre 1978

Rina Lasnier

URI : https://id.erudit.org/iderudit/200139ar

DOI : https://doi.org/10.7202/200139ar

Aller au sommaire du numéro

Éditeur(s)

Les Presses de l'Université du Québec

ISSN

0318-9201 (imprimé)

1705-933X (numérique)

Découvrir la revue

Citer cet article

Piette, A. (1978). Un homme et son péché : l'innocente avarice ou le masque idéologique. Voix et Images, 4(1), 107-126. https://doi.org/10.7202/200139ar d'utilisation que vous pouvez consulter en ligne.

https://apropos.erudit.org/fr/usagers/politique-dutilisation/ 


\section{Un homme et son péché ${ }^{1}$ :}

\section{l'innocente avarice ou le masque idéologique}

En quoi l'analyse de ce roman qui date, peut-elle être de quelque utilité si l'on tient compte du fait que plusieurs critiques ont tenu à peu près le même discours gravitant plus ou moins autour de l'avarice? C'est précisément l'aspect répétitif de ce discours critique qui incite à la présente démarche. Le fait de polariser l'attention du lecteur sur l'avarice au point qu'il n'y voie autre chose semble constituer le premier aspect idéologique du roman: le rapport à l'avacice masque ainsi les véritables rapports à établir. Deux articles émergent toutefois par leur nouveauté de la critique portant sur Un homme et son péché. Le premier, "Séraphin ou la dépossession " de Pierre Desrosiers ${ }^{2}$, est une approche sociocritique du texte inspiré de la méthode de Goldmann. Je m'y référerai à l'occasion au cours de cet article : il a le mérite d'ouvrir la voie, même si parfois des éléments importants du texte y sont négligés ou, ce qui est plus grave, mal interprétés. Je mentionne le second, encore qu'il concerne moins le projet envisagé ici : Gérard Bessette, dans "Autour de Séraphin ${ }^{3}$, a procédé à une approche psychocritique du texte, analysant divers aspects de la sexualité trop peu connue de Séraphin.

L'objectif poursuivi dans le présent article est de montrer comment ce texte est idéotogique ou mieux de quelle façon l'idéoloqie terrienne s'y manıteste. Hon entreprise sera donc différente de celle de Desrosiers sans que, bten sûr, elles se contredisent : il ne s'agira pas de la mise en rapport d'une structure romanesque et d'une structure sociale (Desrosiers), mais de la mise en rapport d'une idéologie et d'une organisation textuelle particuliere de cette toteotogie. Tl est donc impérieux, avant d'aller plus loin, de donner une définition de l'idéologie. C'est à partir de Marx et de la tradition marxiste que s'est développée la notion d'idéologie. La définition que donne Denis Monière dans son récent ouvrage le Développement des idéologies au Québec peut être retenue:

Une idéologie est un système global plus ou moins rigoureux de concepts, d'images, de mythes, de représentations qui dans une société donnée affirme une hiérarchie de valeurs et vise à modeler les comportements individuels et collectifs. Ce système d'idées est lié sociologiquement à un groupe économique, politique, ethnique 
ou autre, exprimant et justifiant les intérëts plus ou moins conscients de ce groupe. L'idéologie est enfin une incitation à agir dans telle ou telle direction en fonction d'un jugement de valeur ${ }^{4}$.

Quant à l'idéologie agriculturiste, elle a été fort bien analysée par Michel Brunet $^{5}$ à qui se réfèrent plus ou moins explicitement quelques ouvrages ultérieurs sur les idéologies au Québec ${ }^{6}$. C'est à ces quelques ouvrages que l'on se reportera pour la compréhension de la présente analyse.

Reste un problème important: celui de la méthodologie. Depuis Althusser en effet, les textes théoriques sur le rapport entre littérature et idéologies ne manquent pas ${ }^{7}$. Mais toutefois, l'analyse des idéologies de textes particuliers - et surtout pour le genre romanesque - se fait plus rare. Jacques Dubois formule la difficulté de ce type d'analyse de la façon suivante: «Peut-on isoler l'idéologie d'un texte? dans l'affirmative, à quoi d'extérieur au texte taut-il la rapporter ${ }^{\nexists}$ ?" On partira ici d'une analyse textuelle selon la méthode pratiquée par Barthes dans $S / Z^{9}$ : le repérage des différents codes du texte. Les codes culturels, entre autres, sont hautement (ou bassement, si l'on veut jouer sur les mots) idéologiques. Quant au code des sèmes, il permettra de bâtir des systèmes sémiques qui fourniront la clef du modèle idéologique du texte.

Où s'arretera máintēnant l'analyse textuelle puisque la méthode du "pas à pas" ne peut être appliquée à tout le texte? On considérera que le début d'un roman offre la plupart du temps l'essentiel des contenus sémique et idéologique (cela est particulièrement vrai d'Un homme et son péché). Le texte sera découpé en lexies selon la pratique de Barthes c'est-à-dire qu'on n'essaiera pas ici de trouver une solution au problème complexe de la scientificité du découpage. On éliminera cependant de cette analyse, la notion de symbole paraissant extrêmement difficile à cerner doit-on suivre Freud, Jung, Bachelard? - et le réseau symbolique d'Un homme et son péché pouvant être en soi un objet de recherche distinct.

\section{Analyse textuelle des huit premières lexies}

1. Le titre: Un homme et son péché

- Le choix de l'article indéfini est significatif. Pourquoi pas «l'homme» ou «Séraphin Poudrier»? II est important de bien établir dès le départ le caractère particulier de cet homme. Séraphin est marginal sur le plan économique, cela va de soi, mais aussi sur les plans sexuel, social, religieux.' Par sa marginalité il échappe à l'idéologie terrienne, mais c'est précisément pour cette raison que l'idéologie se doit de bien le détacher du modèle terrien en montrant qu'il est l'exception qui confirme la règle (SEM. Particularité, marginalité).

- La particule de coordination «et» réunit normalement des choses ou des êtres semblables. Mais Séraphin ne peut (et ne veut) s'accoupler vraiment qu'avec son péché : "Ce qui arrive est pour le mieux. Je vais reprendre ma vie de vieux garçon. Je vais vivre seul et à mon goût. " (p. 137). (SEM. Insociabilité, misanthropie). 
- Le possessif “son " révèle déjà, d'une certaine manière, le thème du roman. II semble ici répondre à une nécessité grammaticale, mais il répond encore davantage à une exigence romanesque (SEM. Possession).

- II n'est pas fortuit que le titre, par le mot "péché", désigne la force aliénante par excellence de l'idéologie 'terrienne : la religion. Ainsi l'idéologie, malgré ses détours, ne peut s'empêcher de se révéler. (REF. Code moral judéo-chrétien).

- La mention du «péché» de Séraphin est le poinţ de départ d'une énigme: quel est le péché de Séraphin? Mais cette phrase herméneutique semble de courte durée puisque, dès le début du premier chapitre (p. 8 , $2^{\ominus}$ paragraphe), le lecteur est fixé sur la nature de ce péché. Pourtant, tout de suite après, l'énigme apparaît irrésolue à cause de l'insistance du narrateur sur «l'impureté » qui travaille Séraphin et plus loin, par l'équivoque qu'il introduit dans son discours en relançant l'énigme: "Bertine, honnête comme un arbre, ne se souciait pas plus de l'homme que de son péché. Elle était loin de penser d'ailleurs que son corps pouvait exciter son cou$\sin "$ ( $p .65$ ). Ce discours hésitant présente en fin de compte moins d'intérêt pour la narratologie que pour la psychanalyse. Rétrospectivement, c'està-dire après la lecture de quelques pages, la généralité du titre (péché) peut apparaître autant comme un effet de censure vis-à-vis de la sexualité que comme un élément énigmatique (HER. Énigme 1 - thématisation).

2. L'incipit: "Tous les samedis, vers les dix heures du matin, la femme à Séraphin Poudrier lavait le plancher de la cuisine, dans le bas côté." (p. 7)

- Dans Un homme et son péché, les rites foisonnent. Cela révèle à la fois l'influence de la religion et le mode de vie terrien où prédomine la répẻtition des mêmes gestes. Ainsi, même le lavage đu plancher est soumis à un protocole: “Tous les samedis, vers les dix heures ou matin (SEM. Ritualisme 8 REF. Mode de vie terrien).

- Nous ne connaissons pas encore Donalda : elle n'est mommée qu'en référence à son mari. Si cette réduction traduit bien la dépendance de Donalda vis-à-vis de Séraphin, elle reflète aussi, au niveau socio-culturel, l'infériorité de la femme mariée qui non seutement perd son nom, mais encare son prënom. En effet, te texte trouve son alibi dans la langue elle-même : lexpression «la femme" était courante dans les campagnes. Mais le texte du même coup fournit un alibi à l'idéologie: Donalda est une exception, elle s'inscrit dans la marginalité même de Séraphin.

Comme par hasard, la première apparition de Donalda dans le texte la montre lavant le plancher. Cette activité, dévalorisante pour une idéologie qui distingue les tâches nobles et les tâches moins nơbles (pour ne pas dire triviales accuse davantage l'infériorité du personnage. Enfin, cet être inférieur lave le plancher dans le bas côté, lieu où se fát Je travail de majson. La suiţe du texte (p. 23) révélera que Donalda n'eșt pas autorisée à se rendre seule dans le haut côté : le haut côté est le lieu des circonstances exceptionnelles, mais surtout il est réservé à Séraphin, c'est le lieu de ses 
«plaisirs ». Déjà cette lexie prépare à la dichotomie travail/plaisir sans qu'on le devine, déjà s'esquisse le partage des sexes, des fonctions, des domaines dans la maison de Séraphin :

$\begin{array}{lll}\text { Femme } & \text { Homme } \\ \text { Travail } & / & \text { Plaisir } \\ \text { Bas côté } & / & \text { Haut côté }\end{array}$

(SEM. Infériorité, dépendance - REF. Statut inférieur de la femme).

- Le signifiant du personnage " "Séraphin Poudrier» mérite une attention particulière. Le choix du signifiant apparaît comme une surdétermination. Les Séraphins constituent une catẻgorie d'anges (il y en a neuf) dans la théologie catholique. D'une part, on évoque l'angélisme, le refus du sexe, du corps, ce qui est justement un des traits de Séraphin. D'autre part, l'ange connote la douceur: la langue a consacré cette connotation par l'expression «douceur angélique». Or, le sème de douceur s'applique souvent à Séraphin (surtout pour la voix), mais il s'agit d'une douceur forcée : c'est de l'hypocrisie ("voix mielleuse», p. 28 et 33 - «mentir d'une voix d'ange», p. 49 - «Séraphin, doucereux, hésitant, chafouin», p. 83). Par ailleurs, "Poudrier" (boîte à poudre) rejoint aussi le dernier sens connoté puisque le maquillage connote le monde des apparences, de la fausseté, en même temps qu'il connote le terrestre et s'oppose au Séraphin angélique, céleste. Quant à «Poudrier" (ouvrier qui fabrique les explosifs), il connote, à côté du Séraphin doucereux, la contradiction et la présence des forces antinomiques (la contradiction apparaissait déjà dans l'opposition céleste/terrestre) (SEM. Angélisme, hypocrisie, contradiction).

- Le début du texte fournit le thème (ou le nom) de la séquence du premier chapitre : le lavage du plancher. Mais avant d'en arriver à la scène singulative (c'est le point de convergence de tout le reste), il faut bien donner l'éclairage approprié à cette scène: la scène itérative du début joue ce rôle explicatif, elle rend la scène singulative lisible. Mais par une logique bien propre à la littérature de représentation faite de liens logico-temporels, on en arrive par la suite à la première rencontre des époux et à l'évolution de la relation jusqu'à la fameuse scène. On obtient ainsi la séquence complète (pour le lecteur virtuel) : scène itérative, analepse explicative, scène singulative ${ }^{12}$. II est important de remarquer que la scène itérative joue alors un double rôle : elle prépare à la scène singulative, mais elle sert aussi d'introduction au roman, car il faut quand même, pour la compréhension du roman, mais aussi pour satisfaire aux lois du genre, présenter les personnages (ACT. Laver le plancher - REF. Code du genre romanesque).

3. “On pouvait la voir à genoux, pieds nus, vêtue d'une jupe de laine grise, d'une blouse usée jusqu'à la corde, la figure ruisselante de sueurs, où restaient collées des mèches de cheveux noirs. Elle frottait, la pauvre femme, elle raclait, apportant à cette besogne l'ardeur de ses vingt ans.

D'un geste vif, précis, elle répandait sur le plancher des poignées de sable blanc et, à l'aide d'un bouchon de paille ou de pesat qu'elle 
trempait dans un seau d'eau, elle frottait d'une main vigoureuse jusqu'à ce que le plancher devînt jaune comme de l'or." (p. 7)

- Lorsque le narrateur affirme: «On pouvait la voir», il faut entendre: «je veux vous faire voir». Ainsi, le spectateur présumé est le destinataire du récit: l'indéfini "on" inclut tout lecteur virtuel, mais en même temps, plus subtil que le "vous", il tend à faire oublier sa fonction en faisant croire à la présence d'un spectateur hypothétique. Ce n'est pas en effet le temps présent qui est employé (temps de la lecture), mais l'imparfait, temps qui projette nécessairement le narrataire dans l'univers représenté. La description romanesque classique tend avant tout à la représentation (REF. Code de la description romanesque).

- Cette femme «à genoux" (position de la prière), "pieds nus" vêtue pauvrement, a tous les traits de la Pénitente (le gris rappelle le mercredi des Cendres). La position d'infériorité de Donalda prolonge et explicite la dénomination de la première lexie «la femme à Séraphin Poudrier " (SEM. Infériorité - Ref. Code judéo-chrétien des rites).

- L'ensemble de la lexie connote l'efficacité : Donalda travaille «d'un geste vif, précis", «d'une main vigoureuse". Ces remarques semblent seulement faire partie de l'appareil descriptif et contribuer au portrait de Donalda, mais par rapport à la scène singulative de la fin du chapitre qui est le point de convergence, elles remplissent une autre fonction : elles tracent implicitement le portrait de l'avare, elles en font un être mesquin qui, tout en trouvant «sa femme dépareillée" (p. 11), ne se prive pas pour autant de lui faire des scènes pour des vétilles. Ainsi, les personnages de roman se construisent les uns par rapport aux autres à partir des informations dispensées par le narrateur. Le narrateur d'Un homme et son péché ne se satisfait pas toutefois de ce contrôle indirect sur ses personnages : à l'instar de beaucoup de narrateurs célèbres. il intervient plus directement par des jugements de valeur, comme par cette apposition mélodramatique («la pauvre femme») qui de toute évidence s'adresse au narrataire: Vous devez la plaindre et par le fait même condamner Séraphin (SEM. Efficacité, pitié REF. Code romanesque).

- Même si la pauvreté est ici dénotée, la fin de la lexie vient annuler l'effet de cette dénotation : tout le passage est ébranlé dans ses assises par la comparaison finale. Cela prouve que la figure n'est pas un simple or nement, comme tafttrme une opinion toute faite et bien codée dans l'histoire littéraire, cela prouve aussi que le pouvoir connotatif peut même faire fi du vraisemblable. En effet, qu'est-ce qu'un aplancher jaune comme de l'or "? II est vrai que certains planchers de maisons canadiermes étaient faits de pin jaune. Mais il y a un excès dans la figure, excès qui de toute évidence attire l'attention sur l'or de Séraphin, omniprésent dans le roman (SEM. Richesse).

- L'aspect pénible du travail de Donalda est mis en évidence par un certain nombre de termes: "la figure ruisselante de sueurs», "elle frottait», "elle raclait», "besognait», etc. Le sème de labeur s'attache ici au 
personnage de Donalda, mais il s'avère aussi d'une plus grande portée : il fait partie de la réalité terrienne et du mythe terrien. Les habitants peuvent-ils vraiment faire autre chose? Ou mieux, doivent-ils faire autre chose? II est symptomatique qu'ici l'activité de Donalda produise de l'or (figuré) pour Séraphin : elle participe à la production, mais non au produit, ce que nous révèle le reste du texte. Mais alors la condamnation de Séraphin ne s'explique-t-elle pas surtout par le fait que son attitude met en présence des valeurs que l'idéologie terrienne interdit de considérer. Le travail de l'habitant doit être envisagé par rapport à la terre uniquement, le produit de la terre ne doit en aucun cas exhiber son pouvoir économique (SEM. Labeur - REF. Le travail de la terre).

4. «Depuis l'âge de dix ans que Donalda faisait ce travail, elle en connaissait bien le mécanisme peu compliqué, mais dur. Quand les reins commençaient de lui chauffer, elle se pliait de telle façon que la douleur disparaissait, ou si un genou lui faisait mal, elle le deplaçait un peu, éprouvant tout de suite une sensation de bien-être qui la reposait et qui redonnait à son corps et à son cœur une poussée verticale de sang et de courage. (p. 7-8)

- Le sème de mécanicité qui apparaît dans cette lexie à propos du travail reviendra à la page 10 , mais appliqué directement à Donalda cette fois : «elle devint cette mécanique qui sert à traire les vaches ", etc. On le retrouve aussi à propos des «tableaux» de la nature par rapport auxquels Dieu est le «Divin machiniste" (p. 144). Or, ce sème implique un autre sème, celui d'instrumentalité, très important chez le personnage de Donalda. Cette dernière est un instrument pour Séraphin, la rationalité lui est refusée : aussi, il est logique que ce mécanisme qu'elle «connaissait bıen soit «peu compliqué " (SEM. Mécanicité, instrumentalité - REF. Statut inférieur de la femme).

- Que Donalda éprouvant de la douleur n'ait qu'à déplacer un peu son genou pour que la douleur se convertisse en "une sensation de bien-être" (le terme est tout de même fort!), la psychanalyse appelle cela du masochisme. II est inutile d'épiloguer sur ce trait de Donalda que le reste du roman illustre suffisamment. Il faut par ailleurs observer que le texte montre le corps de Donalda d'abord dans la douleur; la Donalda du «champ de fraises" ne viendra que plus tard. Cet ordre de présentation n'est pas dépourvu de signification. Notons que ce masochisme physique n'est qu'une manifestation particulière des sèmes déjà relevés à propos de Donalda: dépendance, infériorité, instrumentalité. Si l'on était tenté de dissocier cette manifestation de la sexualité de Donalda, l'intratextualité y contraindrait malgré tout; il suffirait de rapprocher ce passage de deux autres passages du roman concernant Séraphin :

qui redonnait à son corps et à son cœur une poussée de sang et de courage (p. 8).

Une curiosité immense, suivie d'une sensation inexprimable, s'emparait de lui, coulait dans tout son être ainsi qu'une poussée de sang neuf et rapide ( $p$. 21). 
II sentait courir dans ses veines un sang neuf et bouillant (p. 64).

Le texte de la page 21 décrit Séraphin jouissant de son or, en des termes qui appartiennent à la littérature érotique. Celui de la page 64 décrit, non plus le substitut érotique de Séraphin, mais sa pulsion sexuelle pour Bertine. Quant au labeur de Donalda «pour oublier la vie», il est présenté comme une sublimation faisant suite au refus de Séraphin de la satisfaire. La parenté stylistique incite donc à regrouper ces trois passages sous le titre : manifestations de la sexualité (ce qui se spécifie en masochisme dans la lexie 4) (SEM. Masochisme).

5. "Comme toutes les choses qu'elle savait, Donalda avait appris à laver un plancher chez ses parents, à l'époque de la colonisation, au Lacdu-Caribou. Et c'était d'une valeur si considérable que le vieux garçon Séraphin Poudrier, dit le riche, l'avait tout de suite remarqué. II lisait dans les gestes. Ses hautes qualités de paysan retors le poussaient à rechercher, dans la femme, la bête de travail beaucoup plus que la bête de plaisir. Comment aurait-il pu hésiter, puisqu'il posséderait les deux?" (p. 8)

- Le texte laisse ici planer une équivoque sur le savoir de Donalda. A propos du lavage du plancher dont on a vu qu'il implique un savoir minimal, le narrateur suggère par le «toutes les choses» que ce savoir n'est pas si limité. Or, à la page 10, le contenu de ce savoir est énuméré: il se réduit à un certain nombre d'opérations techniques. L'allusion de la lexie 5 ne serait-elle pas plutôt une hyperbole? Et cette allusion ne seraitelle pas un effet de l'idéologie qui, en suggérant un savoír considérabte 'chez la femme, veut masquer le caractère mécanique de son champ de compétence?

La suite de la lexie délimite de façon beaucoup plus claire les fonctions féminines: bête de travail / bête de plaisir (de toute façon, la femme est bête!). Cette conception de la femme-instrument est ancienne. En Occident, elle trouve ses fondements chez les Grecs, cela va sans dire, mais aussi dans la pensée judéo-chrétienne inspirée de saint Paul. Qu'on relise, par exemple, ce passage de la première épître aux Corinthiens :

"L'homme, lui, ne doit pas se couvrir la tête, parce qu'il est l'image et le reflet de Dieu; quant à la femme, elle est le reflet de l'homme. Ce n'est pas l'homme en effet qui a été tiré de la femme, mais la femme de I'homme; et ce n'est pas l'homme, bien sûr, qui a été créé pour la femme, mais la femme pour l'homme. Voila pourquoi la femme doit avoir sur la tête un signe de sujétion \#... (11, 7-10)

Ici, l'idéologie trouve encore un alibi par le biais du personnage. C'est en effet Séraphin (marginal) qui voit la femme de cette façon, nous dit le narrateur. Mais par la suite, ce dernier ne remet pas en question l'expression "bête de travail "; le principal crime de Séraphin, aux yeux du narrateur, semble être de négliger la bête de plaisir: «Et en moins de six mois de mariage, elle devint cette mécanique qui (...) enfin, elle devint la femme à tout faire, excepté l'amour» (p. 10). Le narrateur, dans sa conception pour le moins simpliste, laisse entendre que la reconnaissance de la bête de 
plaisir aurait évité à Donalda de devenir une mécanique (SEM. Animalité, instrumentalité, infériorité / supériorité - REF. Statut inférieur de la femme).

- Un certain nombre de sèmes sont connotés dans cette lexie à propos de Séraphin. L'avarice tout d'abord : à la page 7, tout dénotait la pauvreté ; la mention de "Séraphin Poudrier, dit le riche" à laquelle fait signe le mot "valeur" oriente alors la relecture de la page 7 et donne en même temps une réponse partielle (sera-t-elle jamais totale et définitive?) à l'énigme suggérée par le titre. Il y a ensuite la perspicacité : [il] l'avait tout de suite remarqué. II lisait dans les gestes "; mais ces expressions renvoient aussi à l'importance du regard, au voyeurisme de Séraphin (p. 73-74). Quant au mot "paysan ", c'est son seul emploi dans le roman pour désigner Séraphin, mais l'épithète "retors" lui est appliquée comme un correctif : Séraphin appartient à une catégorie particulière, il n'entre pas dans le paradigme général (sa marginalité est d'ailleurs soulignée par le terme de "vieux garçon »). D'autre part, le terme "paysan (ne)" peut désigner d'autres personnages, sans restriction cette fois (Alexis par exemple), mais alors apparaît son caractère d'emprunt à la littérature française : il trahit le colonisé (par la culture). Le roman de Grignon, pour les niveaux de langue, représente un compromis entre le français de la littérature et les expressions québécoises (pour l'effet de réel) (HER. Énigme I - Réponse partielle SEM. Avarice, perspicacité, voyeurisme, ruse, marginalité - REF. La culture française).

6. «II connut Donalda, enfant. II la convoitait depuis le jour où il l'avait rencontrée dans un champ de fraises. Elle s'était assise près de lui et il avait été frappé par la blancheur de ses bras et par la fermeté de sa poitrine, si opulente pour son âge. II l'aimait. » (p. 8)

- L'inceste est connoté. Toutefois, ce que la seule lexie donne à lire est la pédérastie ${ }^{13}$ de Séraphin. Le mot "enfant" (non "adolescent") ne laisse pas d'équivoque là-dessus; il est d'ailleurs mis en évidence par la virgule qui n'est pas absolument requise dans ce cas. Si on parle alors d'inceste, c'est à cause de l'intratexte: la relation père-fille (Séraphin-Donalda) se manifeste dans la suite du roman, Bessette l'a déjà prouvé de façon convaincante ${ }^{14}$ (SEM. Inceste).

- Les fraises comportent certaines connotations. Le code judéo-chrétien inspiré de la Genèse a vu dans le fruit un plaisir défendu et l'a associé à la femme. Cette connotation est corroborée par la lexie suivante. Quant à la psychanalyse, elle voit dans les fruits un symbole possible des seins (ou des fesses ${ }^{15}$ ), ce qui serait confirmé par la suite du texte : «la fermeté de sa poitrine". De toute façon, le motif des fraises est récurrent. Séraphin, à la page 111, est victime d'une hallucination: Donalda lui apparaît avec «un chapeau de paille rempli de fraises" (le chapeau de paille utilisé par Donalda pour laver le plancher, au chapitre premier, doit donc être associé à la bête de plaisir plutôt qu'à la bête de travail : peut-être alors la colère de Séraphin s'explique-t-elle?). Alexis, à la page 128, rêve à Donalda «qui présentait sa bouche de fraise au miel du soleil ». Cepen- 
dant, le motif des fraises semble inscrit dans un réseau plus vaste impliquant le sème de rougeur. Pourquoi Lemont est-il roux (le narrateur ne mentionne jamais la couleur de cheveux des hommes), Lemont dont la spécialité est bien reconnue par le texte ("le monstre d'impureté ") ? Pourquoi, juste après qu'Arthémise joue avec sa croix "qui tombait sur sa poitrine toujours en mouvement", Alexis, l'homme à femmes, entre-t-il "le visage rouge, le cou rouge, les mains rouges et soufflant très fort " (p. 119)? Pourquoi Alexis (encore) pousse-t-il "ce cri qui sortait tout rouge» (p. 179) ? Ces étrangetés textuelles suggèrent un rapport entre la rougeur et la sexualité : sans doute le rouge connote-t-il l'attrait sexuel (objectif ${ }^{16}$ ) SEM. Attrait - REF. Code moral judéo-chrétien).

7. "Il se laissa d'abord entraîner par le fleuve de l'impureté dont il ne chercha jamais à découvrir la source. Puis, peu à peu, il se fit à l'idée qu'elle pourrait devenir sa femme. Quand la petite eut vingt ans, il l'épousa. II en avait quarante. Les troubles de la chair qu'il combattait depuis tant d'annees l'envahissaient maintenant ainsi qu'une crue prodigieuse de limon. Mais Séraphin ne se laissa point attendrir comme un fol, ni par le cceur, ni par les sens. II se rendit compte avec une précision d'usurier que s'il se laissait aller à la passion de la chair, la petite Donalda Laloge finirait par lui coûter les yeux de la tête et lui mangerait jusqu'à la dernière terre du rang. Il lutta tant et si bien, de nuit et de jour, qu'il fit de sa femme moins qu'une servante : pas autre chose qu'une bête de somme. » (p. 8-9)

- Cette longue lexie gravite autour d'un point central : la résistance de Séraphin à ses pulsions. Très significative, de ce point de vue, la récurrence du verbe "se laisser " : "se laissa d'abord entraîner", " ne se laissa point attendrir», "s'il se laissait aller". Dans le même sens, il faut lire "il se fit à l'idée" ainsi que les verbes "combattait " et "lutta". II est possible de donner un nom plus spécifique à cette résistance de Séraphin si on la met en rapport avec son avarice : la rétention. Séraphin retient ses pulsions sexuelles comme il retient l'argent et les gages de ses débiteurs. On peut même ajouter que cette connotation rejoint le besoin du pouvoir chez Séraphin. II n'y a pas là en effet qu'une domination exercée sur Donalda («pas autre chose qu'une bête de somme»): plus loin dans le texte, ne lit-on pas qu'il «tenait tout le monde dans sa main" (p. 56) ? Ainsi convergent, chez Séraphin, le sexuel, l'économique et le politique sous la forme de la rétention ${ }^{17}$ (SEM. Rétention, supériorité).

- Les tendances pédérastiques et incestueuses de Séraphin sont ici bien soulignées par la mention de la différence d'âge entre les deux époux de même que par l'expression répétée ala petite". Cela pourrait rendre compte d'une phrase apparemment mystérieuse : «Puis, peu à peu, il se fit à l'idée qu'elle pourrait devenir sa femme. » On se demande comment la perspective du mariage ne suffit pas à éliminer d'un coup la résistance de Séraphin puisqu'elle correspond aux normes voulues par la société et la religion. Mais si ce que ressent Séraphin pour Donalda trahit sa fixation œdipienne, on comprend alors que le mariage ne régularisera rien (SEM. Inceste). 
- Le narrateur présente ici ce qu'on pourrait appeler la genèse de l'attitude de Séraphin vis-à-vis de Donalda. A l'origine de son attitude répressive envers sa femme se trouve la répression qu'il a dû exercer à l'endroit de ses pulsions sexuelles. Les termes de combat «combattait " et «lutta" évoquent le code moral judéo-chrétien à propos des instincts, surtout de l'instinct sexuel : c'est un code marqué par la culpabilité, de là les combats épiques qu'il suscite. Mais une fois de plus, c'est la marginalité de Séraphin qui donne le change. Cette culpabilité morbide est celle d'un névrosé, elle ne relève pas d'une idéologie (c'est ce qu'on voudrait nous faire croire, l'avarice servant ici de caution!). Toutefois, la distance du narrateur vis-à-vis de son personnage n'est pas bien grande. II semble contaminé par la culpabilité de Séraphin puisqu'il parle du "fleuve ${ }^{18}$ de l'impureté", des "troubles de la chair", de la "crue prodigieuse de limon", de "la passion de la chair». Ou plutôt ne serait-ce pas que Séraphin et le narrateur se réfèrent au même code? (REF. Code moral judéo-chrétien).

8. «Cette paysanne, fraîche comme un pommier en fleurs, prédestinée aux adorables enlacements, assaillie des désirs que l'invincible atavisme faisait croître autour d'elle comme en un printemps sans fin, ne connut jamais les joies de la charnalité. Elle passa, sans transition, du soir des noces à la vie amère, cassante et matérielle du ménage, sans avoir même éprouvé la sensation d'un baiser lent et profond.

Une fois, une seule fois, Séraphin la posséda brutalement, mais refusa net de lui faire un fils qu'elle désirait avec tant d'amour de par l'hérédité la plus lointaine.

- Je n'aime pas les enfants, avait-il dit, avant de s'endormir. Dans une autre circonstance, il s'était livré :

- Tu sais, ma fille, que des enfants, ça finit par coûter cher. » (p. 9-10)

- Il y a un aspect idyllique dans le tableau brossé ici par le narrateur. On y retrouve, à propos d'une jeune fille, l'arsenal des comparaisons les plus stéréotypées. La nature y est présente comme ornement du discours, mais à bien y réfléchir, on constate qu'elle y joue un rôle plus fondamental que le seul enjolivement. «Pommier en fleurs" et "printemps sans fin " soulignent le rapport étroit qui existe entre l'homme et la nature. Tous les deux semblent entraînés par la même force, soumis à la même nécessité («prédestinée», «invincible atavisme»). Dans cette optique, la nature apparaît comme un guide ou un modèle pour l'homme. Cette description prépare donc et condamne implicitement la transgression de Séraphin (p. 10) qui ne se soumet pas aux lois naturelles. Séraphin n'est pas plus à l'écoute de la nature lorsqu'elle parle de Dieu (p. 144-146). II se maintient en dehors du cycle naturel (il retient son sperme) et y maintient Donalda : ils sont donc voués ensemble à la mort. Pourtant "l'atavisme " de Donalda fait signe à celui d'Alexis (p. 45) qui, lui, est inscrit dans la norme des choses. Donalda est donc victime de la transgression de Séraphin, ce que la présente lexie illustre bien (SEM. Nécessité, transgression, rétention - REF. Code de la description romanesque). 
- Il est temps maintenant de scruter cette nature que le texte prête à Donalda. Que l'on considère la société contemporaine à la parution du livre ou celle qui correspond à l'époque représentée dans le roman, est-il vraisemblable de dire d'une femme qu'elle est "prédestinée aux adorables enlacements, assaillie des désirs»..., qu'elle "se roulait dans le lit conjugal". Ne serait-ce pas plutôt l'effet d'une projection de narrateur (d'auteur!) masculin? La fonction idéologique de pareils énoncés n'est-elle pas encore une fois d'occulter l'extrême état d'inhibition sexuelle qui était le partage des femmes (des hommes aussi, quoiqu'à un degré moindre) ? On semble nous dire ici : le sexe était naturel pour nos paysans, les anomalies ne se trouvaient que chez les Séraphins ${ }^{19}$ (REF. Code moral de la sexualité).

- Il est pour le moins étonnant que le sexe de l'enfant que Donalda désire soit déterminé avant même sa conception. Cela se comprend si l'on considère les impératifs de la vie terrienne (la succession de la terre), ce que le texte souligne indirectement (“de par l'hérédité la plus lointaine»). Mais il y a une autre raison à cela, raison intratextuelle: Donalda fait partie du réseau incestueux que l'on a déjà à propos de Séraphin. Elle éprouve donc un désir comparable à celui de Séraphin. II est remarquable aussi que Donalda qui n'ouvre jamais la bouche ait osé exprimer son désir làdessus : la procréation répond-elle à une loi si sacrée? Sur ce point, on retrouve l'idéologie la plus traditionnelle de la sexualité, idéologie dictée par l'Église : la sexualité doit avoir pour fin la procréation (SEM. Inceste REF. Code moral chrétien, la vie terrienne).

\section{Analyse de l'idéologie}

II serait apparu plus logique que cette analyse textuelle se prolongeât jusqu'à la fin de l'analepse explicative: “Et la pauvre bête de se tuer lentement " (p. 11). Toutefois, la portion de texte analysée est assez représentative de l'ensemble du roman et contient les éléments essentiels à l'analyse de l'idéologie (la pertinence de cette affirmation ne se révélera au lecteur de cet article qu'au terme de l'opération). De la même façon, il aurait été intéressant d'analyser deux autres segments auxquels on se référera au cours de l'analyse de l'idéologie: le portrait d'Alexis (p. 45) et celui de l'abbé Raudin (p. 91-93). Ces deux personnages jouent un rôle primordial dans l'idéologie du roman, mais les sèmes qui les caractérisent sont contenus au moins implicitement dans les premières pages analysées.

A ce stade de la présente étude se pose la question de la procédure. Tout au cours de l'analyse précédente, il a été question d'idéologie, de fragments d'idéologie disséminés dans le texte. Ces observations particulières seront d'une grande utilité, elles permettront de voir dans quelles directions il faut maintenant chercher. Mais l'objectif sera alors plus vaste puisqu'il visera la cohérence globale du roman. Dans la perspective d'une analyse de l'idéologie qui sous-tend Un homme et son péché, les codes actionnel et herméneutique apparaissent d'un intérêt négligeable. II est clair 
que ce type de roman repose tout entier sur ses personnages, que par conséquent le code des sèmes y est un élément fondamental, tandis que les différents codes culturels ou codes de références servent de balises en établissant plus directement le rapport avec un hors-texte. Mais de tous les sèmes relevés à propos de Séraphin et Donalda, que retenir? II est ici opportun d'avoir recours à la notion de pertinence, de ne pas perdre de vue l'objectif final: l'analyse de l'idéologie. Tel sème, l'inceste par exemple, serait pertinent à l'approche psychanalytique du texte, mais ne serait pas ici d'un grand secours.

Ce cadre opératoire étant délimité, on retient trois sèmes principaux. Le sème de rétention tout d'abord, puisqu'il est au centre du personnage de Séraphin, qu'il explique, entre autres choses, sont attitude vis-à-vis de son travail et du produit de son travail. En second lieu, le sème d'infériorité (supériorité ${ }^{20}$ ) relevé à propos de Donalda puisque ce sème, tout en s'attachant à un individu dans le présent contexte, peut aussi concerner un rapport de classe. Enfin, le sème de dépendance relié au précédent, mais sous l'angle plus spécifique du besoin. Si maintenant on tient compte du reste du roman, entre autres des deux passages évoqués précédemment à propos d'Alexis et de l'abbé Raudin, on constate qu'il ne s'agit plus de sèmes, mais vraiment de couples oppositionnels : rétention / don (avarice / prodigalité représentant des manifestations particulières de cette opposition), infériorité / supériorité, dépendance / indépendance. II faut examiner chacun de ces couples oppositionnels et voir de quelle façon se répartissent, sous ce rapport, les différents acteurs du roman.

Le pôle de la rétention est évidemment occupé par Séraphin et son double, le beurrier Brassard. Séraphin, sur le plan sexuel, retient ses pulsions, on l'a déjà vu; il ne se soumet même pas à la loi de la procréation à laquelle l'invite le modèle par excellence, la Terre, “l'admirable prodigue " (p. 69). II adopte la même attitude vis-à-vis de la terre qu'il ménage (p. 54, 69). Quant à ses rapports avec les autres, avec l'argent et les biens en général, c'est sans doute ce sur quoi le texte revient le plus souvent. Très révélateur à ce sujet est le passage sur les gages accumulés pour des prêts non respectés (p. 20-21).

Le pôle opposé est dominé par la terre «qui ne demande qu'à donner, donner encore, donner toujours" (p. 69). Ce genre d'énoncé ne surprend pas dans un roman de la terre, mais on n'a peut-être jamais mis en valeur son rapport avec Séraphin et tiré les conclusions appropriées en relation avec l'idéologie terrienne. De la même façon, le "ministre de la libéralité divine" (p. 93), le curé Raudin, représentant de l'idéologie religieuse, n'a pas été suffisamment identifié comme une des figures antithétiques de Séraphin : "ll eût donné sa soutane pour vêtir les enfants des bois ${ }^{21}$ ". (p. 92). Toutefois, sous l'aspect de la récurrence du sème, Alexis plus que tous les autres représente le don. Contrairement à son cousin, il est d'une fécondité étonnante: «Père de huit enfants" (p. 45). Quant à la terre, il semble l'exploiter le plus possible: «tout ce qu'il arrachait au sein de sa vieille terre" (p. 45). Et de même que pour Séraphin, l'aspect central du 
personnage d'Alexis concerne son rapport avec les autres (Séraphin surtout) et avec l'argent. II est celui qui rend constamment service à Séraphin : il va chercher le docteur, fournit l'aide de Bertine ${ }^{22}$ et d'Arthémise, apporte le lait chez le beurrier, tire la vache jersey de l'eau, etc. Les autres acteurs qui occupent ce pôle sémique ne laissent aucun doute sur l'interprétation qu'il faut donner à la serviabilité d'Alexis même si le texte n'en fait pas état: Alexis s'inscrit dans la ligne de la charité chrétienne (l'abbé Raudin); mais il est aussi à l'image de la terre: "prodigue" (l'emploi du même lexème souligne sans équivoque le rapport que le texte établit implicitement). Du même coup, le fonctionnement économique d'Alexis entre en opposition totale avec l'avarice de Séraphin.

Le couple sémique supériorité / infériorité est illustré dès le premier chapitre par les rapports de Séraphin et Donalda. L'analyse textuelle a déjà amplement révélé ces rapports. Séraphin tient le même rôle dans la séquence de l'emprunt de Lemont. Enfin, un passage capital sur la position dominante de Séraphin vis-à-vis de la majorité des gens du comté mérite d'être cité :

C'est certain, comme la lumière de Dieu nous éclaire, qu'il n'y avait pas un homme dans le comté qui «valait" plus que lui. Lui, Séraphin Poudrier, petit cultivateur, petit prêteur de rien du tout, mais qui tenait tout le monde dans sa main : monsieur le maire, monsieur le docteur, monsieur le député, tous les cultivateurs, depuis le plus gros jusqu'au plus petit. Peu d'hommes mangeant et ayant besoin d'un gîte qui ne lui devaient de l'argent. II était le possesseur et le maître. (P. 55-56)

Ce passage ${ }^{23}$ indique bien les liens du politique et de l'économique chez le personnage de Séraphin. Le texte parle de tout le monde»; on a dit plus haut : "la majorité des gens". Cela peut paraître une mauvaise lecture du texte, mais à bien le considérer, le passage révèle deux omissions. Tout d'abord, dans la liste des notables de la place, le curé ne figure pas. En second lieu, il est dit : «Peu d'hommes... qui ne lui devaient de l'argent” et par ailleurs, Alexis qui a des dettes (p. 45) n'est jamais mentionné comme un des débiteurs de Séraphin. Le texte semble donc vouloir soustraire Alexis au modèle général de relations qu'a Séraphin avec les autres. Le seul aspect où Alexis paraît en position d'infériorité vis-à-vis de Séraphin résiderait dans son attitude empressée, presque servile, à secourir Séraphin. Mais ce trait est largement compensé par les autres qualifications et fonctions d'Alexis. Somme toute, on peut dire que l'abbé Raudin et Alexis n'entrent pas dans la catégorie sémique dominant / dominé en rapport avec Séraphin.

Cela n'empêche pas Alexis d'être en position dominante en d'autres lieux du texte alors que Séraphin n'est pas concerné directement: «En ce moment, fier, le torse cambré, beau, il dominait la plaine et la rivière ${ }^{24}$ " (p. 61). Alexis se doit de dominer la nature, non seulement à cause de sa fonction de mâle dans tout le roman, mais aussi pour des raisons idéologiques : comment ce «paysan par atavisme» pourrait-il apparaître écrasé par les éléments sans dévaloriser la fonction sacrée qu'il doit représen- 
ter? En même temps, cette vision de l'homme correspond à la représentation anthropocentrique de l'univers dont l'origine se situe dans la Grèce antique et qui a pesé sur tout l'Occident depuis.

Un troisième couple sémique se manifeste dans le texte: indépendance / dépendance. C'est de ce point de vue surtout qu'il faut situer Alexis par rapport à Séraphin. D'une certaine façon, ce couple sémique rejoint le précédent, mais il exprime moins un rapport hiérarchique entre deux personnages. C'est une question de nuance. Ce couple sémique est centré sur un manque, un besoin. Alexis n'attend rien de Séraphin, alors que ce dernier compte sur l'autre chaque fois qu'il ne peut obtenir quelque chose. Le narrateur impute souvent cela au désir de faire des économies, mais cette motivation de Séraphin n'élimine pas l'autre aspect, celui où il attend d'Alexis qu'il joue le rôle d'un parent (au sens fort de pèremère). Cette relation de dépendance est nécessaire à la réalisation du don chez Alexis, mais elle affiche moins que le couple supériorité / infériorité la hiérarchie des deux personnages. C'est donc par ce biais que le texte peut maintenir une relative cohérence. Quant à l'abbé Raudin, «il secourait les âmes" (p. 91) et "soulageait les misères du corps" (p. 92). Tout le monde dépend de lui pour quelque chose, sauf Séraphin bien sûr. II va de soi que ce dernier n'attend rien du curé matériellement. Quant au spirituel, la réaction de Séraphin qui n'avait pas pensé à faire venir le curé (p. 83) en dit assez long. C'est d'ailleurs ce qu'en pense le curé qui prie pour sa conversion (p. 93).

Au terme de cette analyse des trois couples oppositionnels, il serait opportun de résumer le résultat de l'enquête :

I. Don

Alexis (Bertine)

La terre

Le curé

II. Supériorité

a) Séraphin

b) Alexis

II!. Indépendance

a) Alexis

b) L'abbé Raudin
Rétention

Séraphin (Brassard)

\section{Infériorité}

Tout le monde (excepté Alexis et l'abbé Raudin), plus explicitement Donalda et Lemont ${ }^{25}$

La nature

Dépendance

Séraphin

Tout le monde (excepté Séraphin)

Si on considère la position de l'acteur Séraphin dans le tableau, on y trouve une confirmation de la lecture intuitive du texte : l'opposition de Séraphin à la plupart des acteurs du roman. Cependant, l'analyse effectuée enrichit la lecture intuitive en ce qu'elle précise quels sont les réseaux oppositionnels et quels acteurs, dans chaque réseau, occupent les pôles opposés. Une première observation se dégage de ce tableau, observation 
que l'analyse des huit lexies (en particulier la septième) avait déjà révélée: Séraphin domine la plupart des personnages par sa rétention. Ainsi se trouvent réunis deux sèmes qui constituent l'acteur Séraphin. La distinction freudienne «principe de plaisir / principe de réalité ${ }^{26}$, à laquelle correspond dans le texte la dichotomie "bête de plaisir / bête de travail ", peut servir d'éclairage à la compréhension du fonctionnement de Séraphin. En niant le principe de plaisir, ce dernier est voué à la névrose ou, selon le terme consacré par la psychanalyse (quoiqu'il ne soit pas des plus heureux!), aux perversions sexuelles (cette dernière solution ne vaut que pour la première partie du roman). Restent donc à Séraphin les activités correspondant au principe de réalité. Le produit du travail de Séraphin représente surtout, étant donné ses privations, des biens de consommation pour les autres, biens convertibles en argent. C'est là le point d'application de sa névrose: l'accumulation d'un capital, séparé du produit dont il émane (et a fortiori de la terre), et la fructification de ce capital. Ce type d'activité rend Séraphin tout-puissant ${ }^{27}$. Les valeurs véhiculées par Séraphin peuvent être illustrées par le schéma suivant :

$\begin{array}{lll}\begin{array}{l}\text { Négation du } \\ \text { principe de } \\ \text { plaisir }\end{array} \rightarrow \begin{array}{l}\text { Travail de } \\ \text { Séraphin }\end{array} \longrightarrow \begin{array}{l}\text { Produit } \\ \text { du } \\ \text { travail }\end{array} & \begin{array}{l}\text { Biens de } \\ \text { consoma- } \\ \text { tion pour } \\ \text { les autres } \\ \text { (le bois } \\ \text { entre } \\ \text { autres) }\end{array}\end{array}$

Mais alors, peut-on se demander, y a-t-il une restriction au pouvoir de Séraphin? II ne faut pas oublier que, dans le deuxième réseau, l'abbé Raudin et Alexis constituent l'exception qui est soustraite au pôle de l'infériorité. II ne faut pas oublier non plus le troisième réseau. C'est par ce biais que l'idéologie récupère Séraphin malgré lui : il est dépendant d'Alexis et ce dernier, de par son acceptation du rôle du curé, est lui-même dépendant du curé. Il apparaît donc maintenant évident que, malgré son propos incessant sur l'avarice, le texte veut suggérer un autre modèle, le modèle terrien. On pourrait formuler ce modèle de la façon suivante:

Finalité spirituelle

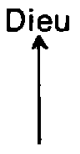

prodigue $=$ pauvre

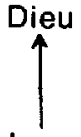

Richesses de la nature

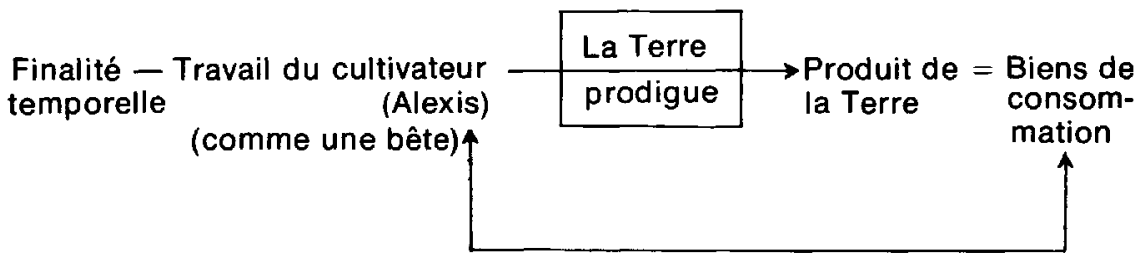


La terre est ici idéalisée et présentée comme paradigme (la terre prodigue ${ }^{2 B}$ ). Mais alors que les richesses de la nature sont inépuisables et réfèrent nécessairement à Dieu (la preuve de l'existence de Dieu, p. 145146 et 163), celles du cultivateur s'épuisent et l'obligent à travailler a comme une bête" pour faire de nouveau fructifier la terre afin d'éviter la famine. Ainsi, ce que le texte ne dit pas, "prodigalité" ne signifie pas la même chose pour la terre et pour le cultivateur. Ce dernier, s'il est prodigue, est nécessairement pauvre. C'est là qu'intervient la morale des Béatitudes, incarnée par Donalda, morale de l'abbé Raudin qui est centrée sur les "richesses spirituelles" et dont la formule inversée est la suivante: l'argent rend malheureux («ce Séraphin toujours fouaillé, dans sa richesse, par les pires tourments $")$. On est tout de même loin de l'adage anodin: "l'argent ne fait pas le bonheur". Ici, c'est la pauvreté qui semble faire le bonheur, si on en juge par le.sourire inébranlable d'Alexis. Mais les autres habitants, "tous ces gens misérables" (p. 193), sourient-ils aussi? Le texte se garde bien d'en parler, comme il se garde bien de faire quelque allusion aux causes politico-sociales de la misère des cultivateurs. L'idéologie agriculturiste des années 1930 avait beau jeu puisqu'elle utilisait comme point de comparaison l'extrême misère des travailleurs québécois des villes.

On peut donc dire qu'Alexis est le représentant principal du modèle terrien dans Un homme et son péché et quoi que les critiques aient pu en dire ${ }^{29}$, ce modèle n'est pas plus contesté que dans les romans de la terre qui l'ont précédé (à l'exception de la Scouine). Ce ne sont que les détours pris par l'idéologie qui font la différence (à ce sujet, l'avarice de Séraphin constitue l'écran majeur qui masque l'idéologie terrienne). Une des meilleures preuves du rôle d'Alexis, c'est l'attitude récupératrice et partiale du narrateur à son égard :

courant souvent la galipote et dépensant comme un fou... II vivait heureux malgré ses dettes (p. 45).

Tu comprends, je serais ben venu hier, mais j'ai été retenu au village.

La vérité, c'est qu'il avait bu jusqu'à se saouler, le pauvre homme (p. 81).

On l'écoutait avec plaisir car il n'était point menteur... On aimait du reste ce bon vivant, grand cœur, bon homme et si honnête (p. 130).

On voit par là comment Alexis sert de support idéologique dans le roman, cela même au prix des incohérences relevées plus haut. Puisque Alexis est nécessaire à l'idéologie terrienne dans ce texte, on l'exonérera de tout blâme même s'il va à l'encontre des normes morales de cette même idéologie.

Séraphin au contraire se doit d'être condamné par rapport au paradigme Terre-Alexis. II est transgressif sur tous les plans : religieux, moral, sexuel, économique et politique. Le texte le condamnera et de plusieurs façons, par une surdétermination idéologique. Il ne suffira pas de l'opposer à Alexis, à la Terre et à l'abbé Raudin, il faudra de plus le faire périr par le feu (les flammes de l'enfer). II faudra encore - on l'a déjà souligné - le 
récupérer dans le modèle terrien (nouvelle incohérence de l'idéologie qui tantôt condamne et tantôt récupère le marginal) par le biais du couple oppositionnel indépendance/dépendance.

Au terme de cette longue étude, il convient de s'interroger sur l'obsession du narrateur vis-à-vis de l'avare Séraphin et sur la figure mythique que produit cette obsession (l'aspect mythique du personnage est confirmé par sa survie au Québec jusqu'à nos jours - popularité de l'émission radiophonique, puis du téléroman, réédition du roman, acception du mot "séraphin" dans le langage québécois - alors que le contexte socioculturel s'est transformé). Si l'on postule que le mythe remplit une fonction idéologique, il faut alors expliquer quels mécanismes opèrent dans ce mythe-ci.

On connaît l'origine de l'idéologie terrienne chez nous: la domination politique, économique et sociale des Anglais et, à la suite de la rébellion de 1837, la réaction de la majeure partie de l'élite petite-bourgeoise, entre autres du clergé, qui a favorisé l'idéologie de la collaboration. II semble évident, après l'analyse des deux modèles du roman, qu'Un homme et son péché, malgré les apparences et les masques, tient un discours équivalent à celui des autres romans terriens. Le fait qu'il s'agisse des valeurs économiques (l'infrastructure) aurait dû attirer l'attention de la critique, d'autant plus que l'argent y est associé au pouvoir. Mais le texte occulte totalement la vie politique des gens (si on le compare à Trente arpents par exemple) et réduit cet aspect à la névrose du pouvoir d'un individu dont la puissance n'est même pas sanctionnée par une fonction politique légale. II y a toutefois une faille (encore!) dans le texte:

Une fois, les gens furent fort intrigués de le voir entrer à l'hôtel, tiré à quatre épingles, buvant en compagnie de deux étrangers qui riaient, qui parlaient fort et qui payaient souvent la traite, en donnant de grandes tapes dans le dos courbé de l'avare. Que se passait-il ?... Décidément, le prêteur tripotait de grosses affaires. (P. 147-148)

Qui sont ces étrangers? II est très probable, étant donné l'époque, que ces hommes d'affaires étrangers soient anglais (ou peut-être américains). Plutôt que de spécifier, le texte se reporte sur le terme générique à la fois si suggestif et si peu compromettant. II est uțile de mentionner ici que c'est une tendance marquée, dans nos romans terriens, de taire le nom de l'anglais et de lui préférer le terme connotatif d'étranger ("l'étranger" de la Terre paternelle. "Des étrangers sont venus" de Maria Chapdelaine, repris par Menaud, etc.).

Ce qui peut constituer un indice intéressant, c'est que ce passage assimile Séraphin aux deux bourgeois : il est traité par eux d'égal à égal (les tapes dans le dos!); on suggère plus loin qu'ils sont "ses deux associés». Enfin, après son voyage, Séraphin lui-même se sent plus que jamais investi d'un statut social privilégié : il en reconnaît le signe à l'allure de sa maison qui a resssemblait aux habitations des vieilles seigneuries du Québec " et qui "portait un air de bourgeoisie et de confort" (p. 152). Serait-il présomptueux d'émettre l'hypothèse que Séraphin tient la place de l'Anglais 
dans le roman? On lui prête alors tous les défauts susceptibles de le faire condamner par l'idéologie religieuse et morale afin d'avoir le plus de raisons possible de le rejeter. Entre autres, l'avarice de Séraphin permet de refuser les valeurs économiques et en particulier l'argent ${ }^{30}$, ce qui rencontre parfaitement l'idéologie traditionnelle là-dessus (notons que le tabou sur l'argent est très ancien dans la doctrine de l'Église catholique, comme Sartre l'a exposé dans ses Réflexions sur la question juive). Mais l'ambivalence demeure puisqu'on voudrait bien être à sa place: «Tous les esprits rampants en vinrent à admirer l'homme et son péché " (p. 149). Par quoi il appert que le mythe de Séraphin peut encore trouver des assises chez nous.

Revenons, en terminant, à la procédure adoptée pour cette analyse, procédure qui fut d'abord empirique pour être ensuite systématique. La première phase, l'analyse textuelle, peut paraître trop longue et peu économique en rapport avec la deuxième. Je crois cependant qu'elle fournit une prise sur le texte, qu'elle permet de mesurer quels détails forment la trame du texte. C'est de cette façon que la révélation d'une structure globale apparaît moins gratuite. Mais d'ailleurs, existe-il une recette pour l'analyse des textes ${ }^{31}$ ?

Alain Piette

Université du Québec à Montréal.

1. C.-H. Grignon, Un homme et son péché, Montréal, Éditions Alain Stanké, 1976.

2. Parti pris, vol. 4 , numéros 5-6, janv.-fév. 1967, p. 52-62.

3. Une littérature en ébullition, Montréal, Éditions du Jour, 1968, p. 91-107. II faut aussi citer une autre étude psychocritique d'Un homme et son péché : C.M. Gagnon, "Concupiscence et avarice chez Séraphin Poudrier", Voix et images, vol. I, numéro 2, décembre 1975, p. 196-205. Cet article reprend plus ou moins un certain nombre de points majeurs de l'analyse de Bessette. II fournit cependant un élément nouveau et fort important : le rapport de l'avarice avec l'analité.

4. Montréal, Éditions Québec/Amérique, 1977, p. 13.

5. "Trois dominantes de la pensée canadienne-française : l'agriculturisme, l'antiétatisme et le messianisme", dans la Présence anglaise et les Canadiens, Montréal, Éditions Beauchemin, 1968, p. 113-166.

6. Jean-Paul Bernard, les ldéologies québécoises au 19e siècle. Montréal, Éditions du Boréal Express, 1973 et Denis Monière, op. cit. Quant au livre de l'Université Laval, 1974, son introduction fournit des indications intéressantes sur l'idèologie terrienne. Mais le chapitre sur Un nomme et son péché reprent, somme toute, les idees les plus traditionnelles sur ce roman.

7. Entre autres, Théorie d'ensemble, Paris, Éditions du Seuil, 1968.

8. L'Assommoir de Zola: sociêté, discours, idéologie, Paris, Librairie Larousse. 1973, p. 102.

9. Roland Barthes, S/Z, Paris, Éditions du Seuil, 1970.

10. Le rôle du titre dans l'inscription idéologique a été analysé par Jacques Dubois dans l'ouvrage déjà cité. II en est ainsi de l'incipit du roman, comme il apparaîtra dans la suite de cette analyse.

11. Voir à ce sujet $\mathbf{P}$. Hamon, "Pour un statut sémiologique du personnage", Poétique du récit, Paris, Éditions du Seuil, 1977, p. 142 et suiv.

12. J'ai repris ici la terminologie de Gérard Genette dans Figures III, Paris, Éditions du Seuil, 1973.

13. Je redonne au mot sa pleine valeur étymologique, ce qui ne correspond pas à l'usage du français. On pourrait aussi parler dans ce cas de «lolitisme».

14. Gérard Bessette, op. cit.

15. S. Freud, Introduction à la psychanalyse, Paris, Petite bibliothèque Payot, 1975, p. 141. Je dois ici justifier la parenthèse. Cette lexie semble s'expliquer 
par la référence à l'oralité. Toutefois, rien n'est moins clair si l'on considère l'ensemble du texte, ainsi que les écrits de Freud, Ferenczi, Abraham, Jones sur l'analité et ses rapports avec l'avarice. II est étrange que Bessette, dans son remarquable article, n'en ait pas fait état. Gagnon, dans l'article cité précédemment, parle et de l'oralité et de l'analité de Séraphin. Mais alors se pose le problème suivant : y a-t-il projection de l'auteur dans le personnage de Séraphin et si oui, l'oralité et l'analité sont-elles présentes au même niveau? II est possible par exemple que la fixation orale reflète l'auteur et que la fixation anale soit surtout présente à cause du sujet (l'avarice), donc plus superficielle. A l'inverse, la fixation orale peut servir de couverture sociale pour donner le change sur la véritable fixation de l'auteur. De toute façon, ces deux aspects du texte expliquent en partie du moins "l'effet de brouillage " dont parle Bessette dans son article. Les précédentes remarques s'inspirent d'une consultation faite auprès d'un collègue, M. H.-P. Jacques, qui effectue une recherche sur les marques orales, anales, utérines, phalliques de l'écriture, recherche qui déboucherait éventuellement sur une typologie de l'écriture. Je n'entends donc pas donner ici une solution à ce problème qui mériterait un traitement particulier.

16. Qu'on se rappelle le développement déjà fait sur la “poussée de sang». Une étude plus approfondie devrait toutefois être menée dans le but de préciser, si c'est possible, grâce à la méthode psychanalytique, ce que le rouge peut signifier de plus spécifique.

17. Ces remarques sur la rétention fourniraient évidemment des arguments en faveur de l'analité de Séraphin.

18. Cette lexie inaugure un réseau métaphorique où l'eau est associée à la pulsion sexuelle de Séraphin : "fleuve", "source", "crue». En saine logique, le texte associera alors la frustration de Donalda à la soif : "Les premières fois, Donalda se roulait dans le lit conjugal, tandis que l'époux, à ses côtés, dormait comme une bûche. Une soif intense la torturait " (p. 10). On ne voit pas très bien comment la privation sexuelle engendre la soif à moins qu'on ne situe cette phrase étrange dans le réseau évoqué plus haut. La soif de Donalda ne la quittera qu'à sa mort, ce que le texte justifie tantôt par la température chaude, tantôt par la fièvre. Par ailleurs, le feu, pôle antithétique de l'eau, remplira la même fonction : "Et si les flammes de la luxure s'acharnaient sur Séraphin... " (p. 10).

19. Le texte analysé développe davantage le pôle de l'infériorité, mais comme on l'a vu, il implique son opposé, le pôle de la supériorité (Séraphin).

21. II est clair que Pierre Desrosiers, dans l'article mentionné plus haut, expédie trop facilement cet aspect lorsqu'il écrit : "Notons en passant que toute valeur religieuse est totalement absente de l'univers d'Un homme et son péché » (p. 57). J'ai souligné "toute" et "totalement " pour mettre en évidence l'excès de cet énoncé : l'auteur de l'article confond, semble-t-il, le personnage de Séraphin et le roman.

22. Bertine elle-même se situe dans le prolongement d'Alexis (atavisme?): “Elle aurait donné sa vie pour sauver Donalda " (p. 87).

23. Voir aussi le passage où l'on parle de "l'homme puissant, terrible..." (p. 148149), ainsi que l'autre passage où la maișon de Séraphin est mise en relation avec sa position dominante (p. 152).

24. Dans ce passage, Alexis domine le bois (de sa voiture). Comment ne pas voir dans cette figure une association de l'habitant et du bois, produit principal de l'économie canadienne au $19^{\mathrm{e}}$ siècle? Mais la situation socio-historique est ici corrigée par le texte: I'habitant domine le bois, c'est-à-dire l'économie. Par ailleurs, Séraphin utilise le bois pour exploiter les autres.

25. Bertine est la figure renversée de Donalda puisque c'est elle qui domine Séraphin. Mais cette inversion des rôles me paraît concerner davantage la psychanalyse que l'analyse de l'idéologie. De plus, Bertine n'est qu'une étape dans I'histoire de Séraphin: elle disparaît totalement du texte après la mort de Donalda.

26. Freud avait une profonde perception des rapports entre le sexuel et l'économique lorsqu'il écrivait: "La base sur laquelle repose la société humaine est, en dernière analyse, de nature économique : ne possédant pas assez de moyens de subsistance pour permettre à ses membres de vivre sans travailler, la société est obligée de limiter le nombre de ses membres et de détourner leur énergie de l'activité sexuelle vers le travail. " (Intr. à la psych., p. 291). 
27. Pierre Desrosiers, dans son article, a observé avec beaucoup de perspicacité que la domination de Séraphin s'exerce sur les individus et non sur la collectivité.

28. Il est évident que ce paradigme concerne, entre autres choses, la sexualité. C'est l'Église qui prescrivait les restrictions au plaisir sexuel lorsque ce dernier n'était pas relié à la procréation. Mais ici, les limites au principe de plaisir semblent plutôt venir du modèle naturel : c'est la terre qui sert de caution!

29. Par exemple Gilles Marcotte, Une littérature qui se fait, Montréal, Les éditions HMH, 1962: "Comme on le remarquait déjà, à sa parution, le roman «ne contient aucune thèse m; mais il signifie d'autant plus clairement, et plus profondément" (p. 29) ou plus récemment, Mireille Servais-Maquoi, dans l'ouvrage cité: "Si Grignon intègre son cuvre à la lignée des romans du terroir, toujours très en faveur à l'époque, il se refuse à célébrer comme ses contemporains, la terre canadienne sur le mode du lyrisme élogieux. II souhaite au contraire initier son lecteur à une vision du monde paysan dépourvue de la moindre intention idéalisante" (p. 129). II semble que les critiques sont induits en erreur par les marques d'écriture realiste de Grignon.

30. L'hypothèse proposée ici ne peut pas négliger complètement le rôle qu'a pu jouer la crise économique dans la production d'un pareil roman.

31. Cette recherche s'est élaborée dans le cadre d'un cours donné à I'UQAM à I'hiver 1977 et à l'automne 1977. Je veux ici reconnaître l'apport précieux de ces deux groupes d'étudiants : leurs remarques m'ont plusieurs fois permis de nuancer et de réajuster certaines affirmations. 